\title{
Visualizing cadherin intermembrane adhesion assemblies using cryo-electron tomography
}

Micah Rapp ${ }^{1}$, Alex Noble ${ }^{2}$, Lawrence Shapiro ${ }^{3}$, Clint Potter $^{2}$, Bridget Carragher ${ }^{2}$ and Julia Brasch ${ }^{4}$

${ }^{1}$ Columbia University, NEWARK, New Jersey, United States, ${ }^{2}$ New York Structural Biology Center, New York, New York, United States, ${ }^{3}$ Columbia University, United States, ${ }^{4}$ University of Utah, United States

Cadherins are a superfamily of transmembrane proteins that contain extracellular cadherin (EC) domains, $\beta$ barrels connected by linker regions that typically bind calcium ions adding rigidity to their structure (1). Classical cadherins, the most well-characterized sub-branch of the superfamily, are adhesion molecules that form intracellular adherens junctions that are key to the development and maintenance of tissue architecture from epithelia (2) to the central nervous system (3). Classical cadherins mediate adhesion by trans interaction with a cognate cadherin molecule on an opposing membrane surface, the interface of which involves a conserved strand swapping mechanism between the membrane distal EC1 domains (4-6). The two subfamilies of vertebrate classical cadherins - type I and type II - both contain five EC domains, a single-pass transmembrane region and a cytoplasmic domain that interacts with the actin cytoskeleton through $\beta$ - and $\alpha$ catenin, but vary in their expression patterns and the exact details of the EC1 strand-swapping interaction. Type I cadherins, like other adhesion molecules (7), have been shown to form ordered arrays between cell surfaces $(5,8)$ by combining trans interactions with cis interactions between molecules on the same cell surface (Fig. 1A). As expected, mutations in type I cadherins that disrupt trans interactions were found to completely ablate all adhesion, while mutations targeting the cis interface prevent the formation of ordered assemblies in crystal structures, on liposomes and on the cellular level (5). However, whether type II cadherins form ordered assemblies is as yet unknown. Structural characterization of type II cadherins is an important step towards understanding their potential role in downstream signaling or the conveyance of force between opposing cells.

In this work, we have recapitulated the biophysical environment of cadherins on opposed membrane surfaces by tethering purified cadherin ectodomains to synthetic liposome membranes, which are visualized by cryoelectron tomography (cryo-ET). Cryo-ET of cadherins at liposome contact sites reveal that both type I Ecadherin and VE-cadherin, an outlier that has features of both type I and type II cadherins, spontaneously form highly ordered assemblies that are distinct in appearance (Fig. 1B), while type II cadherins-6, -8 , and -10 formed moderately ordered assemblies (Fig. 1C). Tomograms of VE-cadherin contain liposomes that have highly parallel membranes with a strong mid-line density and a consistent membrane spacing of approximately 30nm (Fig. 1B, left panel). Tomograms of E-cadherin show consistent EC domain spacing when the array is viewed from the top (Fig. 1B, right panel). Tomograms of cadherin-6, -8, and -10 show less-ordered protein density but numerous parallel membrane surfaces and midline densities. These data are crucial to understanding the structure and function of these molecules as, unlike type I cadherins, we lack high-resolution crystal structures of full ectodomains containing both trans and cis interfaces that could reveal the nature of these assemblies. Ongoing sub-tomogram averaging of the assemblies formed by VE-cadherin revealed a highly ordered mid-line density and will likely identify cis and trans interfaces in domain-level resolution that will allow for site directed mutagenesis to identify interface contacts that are responsible for assembly formation.

The observed assemblies formed by classical cadherins differ sharply from those previously observed for the clustered protocadherin branch, a family of molecules that contain six EC domains and mediate neurite-neurite recognition in the mammalian central nervous system (9). Unlike the classical cadherins, clustered protocadherins engage in homophilic trans interactions mediated by the membrane-distal domains EC 1-4, as well as promiscuous cis interactions through membrane-proximal domains EC 5-6 (10-13). A crystal structure of full-length protocadherin $\gamma \mathrm{B} 4$ revealed the details of a linear 'zipper' assembly (Fig. 2A), which was confirmed by cryo-ET of $\gamma$ B6 adhered to liposome membranes (Fig. 2B)(14). Given the strictly homophilic 
nature of the trans interaction and the subset of clustered protocadherin isoforms that are expressed in each neuron, this linear assembly provides an elegant chain-termination mechanism that allows opposing neurites to self-avoid.

Taken together, studies of classical cadherins and clustered protocadherins provide insight into the possible roles played by linear and 2D cadherin adhesion assemblies formed between cells, as well as an adaptable platform with which to study adhesive events mediated by other molecules.

A

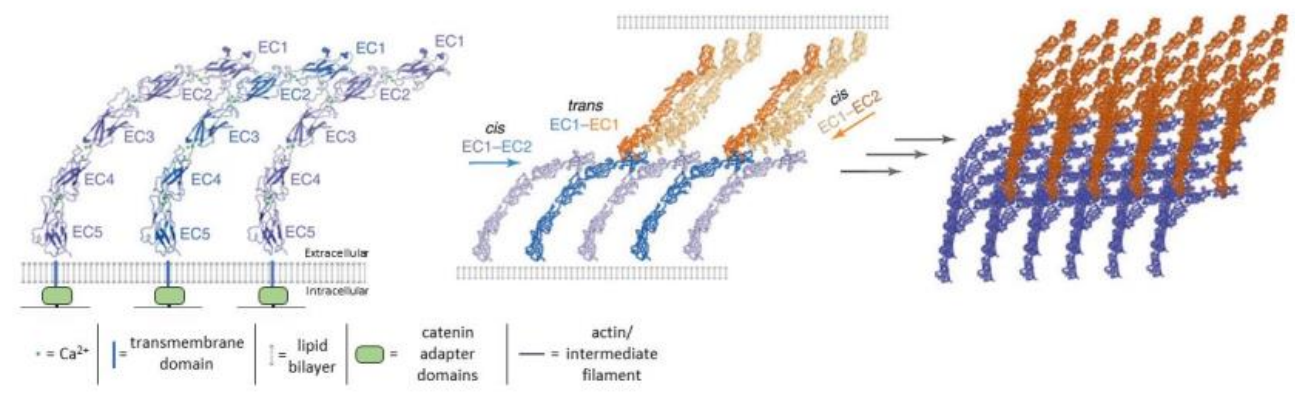

B
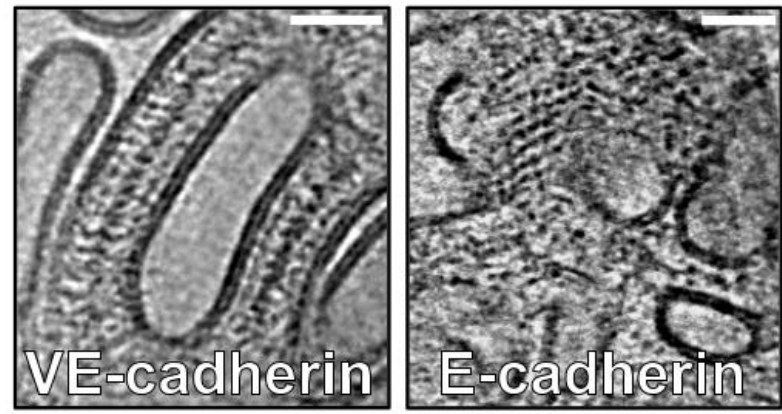

C
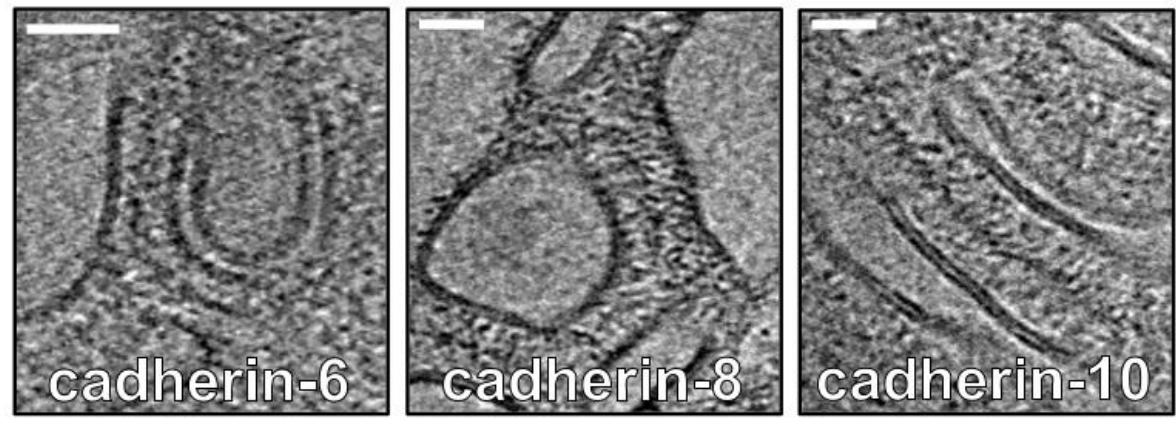

Figure 1. Figure 1. Classical cadherins form 2D lattice-like arrays A) Crystal structure of the five EC domain classical N-cadherin revealing linear cis interactions (left panel) and perpendicular trans interactions (middle panel) that combine to form an ordered 2D array (right panel). Adapted from Brasch et al., 2012. B) Slices of cryo-ET tomograms of VE- and E-cadherin show a high degree of order. VE-cadherin (right panel) 
ectodomains tethered to liposome membranes reveal ordered assemblies at liposome contact sites. E-cadherin tomograms reveal regularly spaced protein density when viewed from the top that matches the arrays observed in crystal structures. Scale bar=35nm. C) Slices of cryo-ET tomograms of type II cadherins- $6,-8$, and -10 taken at liposome contact sites show a moderate level of order with strong midlines, parallel membrane surfaces and consistent intermembrane distance. Scale bar $=35 \mathrm{~nm}$.

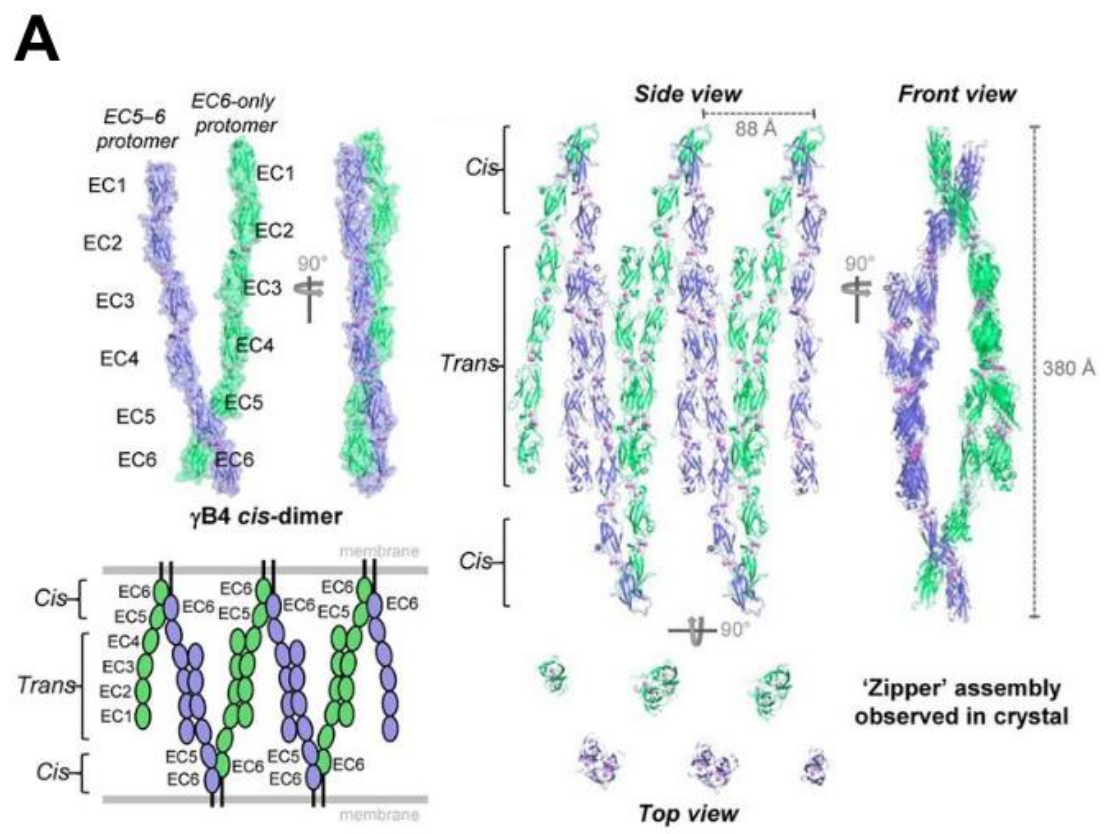

\section{B}
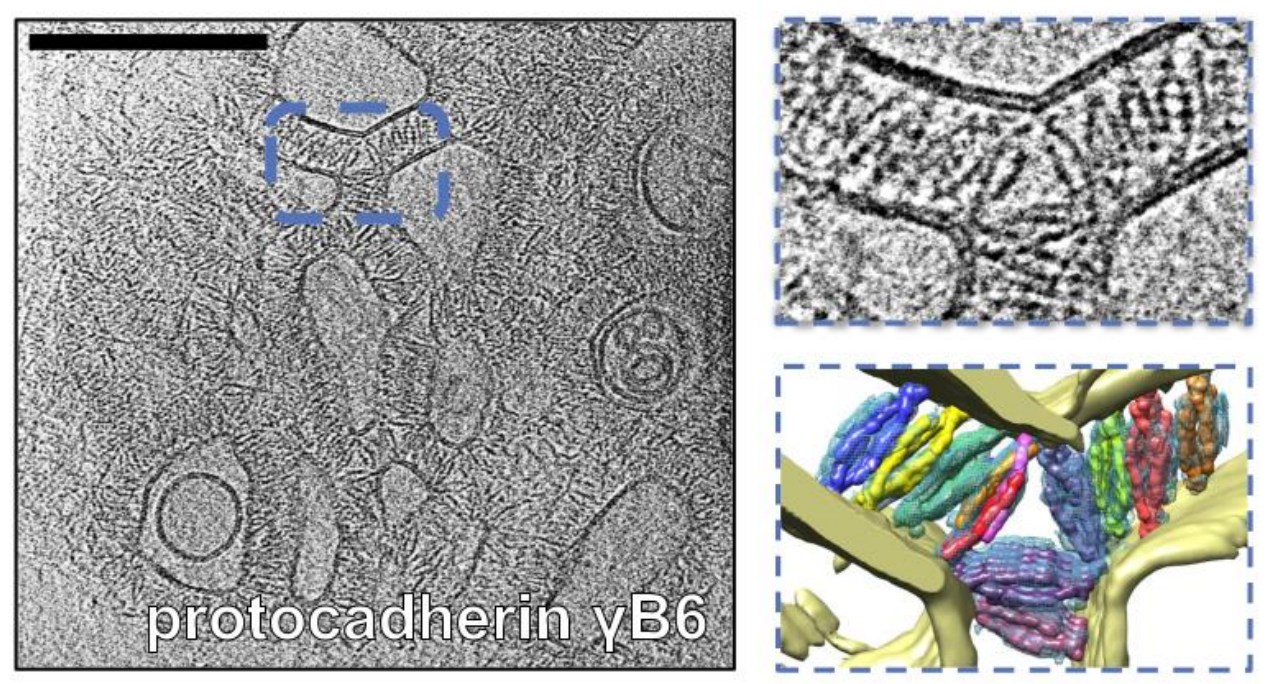

Figure 2. Figure 2. Clustered protocadherins assemble into linear zipper arrays. A) Crystal structure of the six EC domain clustered protocadherin $\gamma \mathrm{B} 4$ showing the promiscuous cis interaction (top left panel) and how it combines with the strictly homophilic trans interaction to form a linear zipper (right panel). B) Slice of cryoET tomograms of clustered protocadherin $\gamma \mathrm{B} 6$ forming ordered assemblies between membranes of coated 
liposomes. The zoomed in view in the top right panel shows clear density for individual EC domains including the cis and trans interfaces resembling those observed in the x-ray structures. The crystal structure of $\gamma \mathrm{B} 4$ was docked into segmented maps revealing close correspondence between zippers observed in the crystal lattice and tomograms (bottom right panel). Scale bar=250nm.

\section{References}

1. P. Hulpiau, F. van Roy, Molecular evolution of the cadherin superfamily. The International Journal of Biochemistry \& Cell Biology 41, 349-369 (2009).

2. M. G. Farquhar, G. E. Palade, Junctional complexes in various epithelia. The Journal of cell biology 17, 375-412 (1963).

3. S. Hirano, M. Takeichi, Cadherins in brain morphogenesis and wiring. Physiological reviews 92, 597-634 (2012).

4. L. Shapiro et al., Structural basis of cell-cell adhesion by cadherins. Nature 374, 327-337 (1995).

5. O. J. Harrison et al., The extracellular architecture of adherens junctions revealed by crystal structures of type I cadherins. Structure 19, 244-256 (2011).

6. J. Brasch et al., Homophilic and heterophilic interactions of type II cadherins identify specificity groups underlying cell-adhesive behavior. Cell reports 23, 1840-1852 (2018).

7. A. R. Aricescu, E. Y. Jones, Immunoglobulin superfamily cell adhesion molecules: zippers and signals. Current opinion in cell biology 19, 543-550 (2007).

8. T. J. Boggon et al., C-cadherin ectodomain structure and implications for cell adhesion mechanisms. Science 296, 1308-1313 (2002).

9. J. L. Lefebvre, D. Kostadinov, W. V. Chen, T. Maniatis, J. R. Sanes, Protocadherins mediate dendritic selfavoidance in the mammalian nervous system. Nature 488, 517-521 (2012).

10. R. Rubinstein et al., Molecular logic of neuronal self-recognition through protocadherin domain interactions. Cell 163, 629-642 (2015).

11. K. M. Goodman et al., Structural basis of diverse homophilic recognition by clustered $\alpha$-and $\beta$ protocadherins. Neuron 90, 709-723 (2016).

12. K. M. Goodman et al., $\gamma$-Protocadherin structural diversity and functional implications. Elife 5, e20930 (2016).

13. K. M. Goodman et al., Protocadherin cis-dimer architecture and recognition unit diversity. Proceedings of the National Academy of Sciences 114, E9829-E9837 (2017).

14. J. Brasch et al., Visualization of clustered protocadherin neuronal self-recognition complexes. Nature 569, 280-283 (2019). 